\title{
SIRT2 protects peripheral neurons from cisplatin-induced injury by enhancing nucleotide excision repair
}

\author{
Manchao Zhang, Wuying Du, Scarlett Acklin, Shengkai Jin, and Fen Xia \\ Department of Radiation Oncology, University of Arkansas for Medical Sciences, Winthrop P. Rockefeller Cancer Institute, Little Rock, Arkansas, USA.
}

\begin{abstract}
Platinum-based chemotherapy-induced peripheral neuropathy is one of the most common causes of dose reduction and discontinuation of life-saving chemotherapy in cancer treatment; it often causes permanent impairment of quality of life in cancer patients. The mechanisms that underlie this neuropathy are not defined, and effective treatment and prevention measures are not available. Here, we demonstrate that SIRT2 protected mice against cisplatin-induced peripheral neuropathy (CIPN). SIRT2 accumulated in the nuclei of dorsal root ganglion sensory neurons and prevented neuronal cell death following cisplatin treatment. Mechanistically, SIRT2, an NAD+-dependent deacetylase, protected neurons from cisplatin cytotoxicity by promoting transcription-coupled nucleotide excision repair (TC-NER) of cisplatin-induced DNA cross-links. Consistent with this mechanism, pharmacological inhibition of NER using spironolactone abolished SIRT2-mediated TC-NER activity in differentiated neuronal cells and protection of neurons from cisplatin-induced cytotoxicity and CIPN in mice. Importantly, SIRT2's protective effects were not evident in lung cancer cells in vitro or in tumors in vivo. Taken together, our results identified SIRT2's function in the NER pathway as a key underlying mechanism of preventing CIPN, warranting future investigation of SIRT2 activation-mediated neuroprotection during platinum-based cancer treatment.
\end{abstract}

\section{Introduction}

Chemotherapy-induced peripheral neuropathy is one of the most common causes of chemotherapy dose reduction and discontinuation, placing limitations on the life-saving therapy that chemotherapy provides (1-3). The systemic neuronal toxicity produced by antineoplastic agents, such as platinum-based agents, is known to cause diffuse, bilateral degenerative changes in peripheral sensation that lead to an altered perception of cold, heat, and pain. Clinically, cisplatin-induced peripheral neuropathy (CIPN) presents as pain that is burning, shooting, or electric-shock-like, causing a major, and often permanent, impediment to quality of life in cancer patients $(1,4,5)$. Because cancer prevalence continues to increase, so does its treatment with chemotherapy, and CIPN is now an urgent, unresolved medical problem for which there are no effective treatments or preventive measures available $(1-3,6)$.

Current understanding of the mechanisms responsible for CIPN is extremely limited. Even though many working models have been proposed (e.g., demyelination of sensory neurons, vitamin alteration, axonal transport, DNA- or RNA-damage-induced neuronal cytotoxicity, cytokine-mediated inflammatory response, etc.), the exact mechanisms of CIPN remain to be elucidated. In addition to peripheral neurons, other cells implicated in the development of CIPN include Schwann cells, Langerhans cells, and macrophages. Antineoplastic treatments have been shown to

Authorship note: MZ, WD, and SA contributed equally to this work. Conflict of interest: The authors have declared that no conflict of interest exists. Copyright: @ 2020, American Society for Clinical Investigation.

Submitted: June 28, 2018; Accepted: February 26, 2020; Published: May 4, 2020. Reference information: / Clin Invest. 2020;130(6):2953-2965.

https://doi.org/10.1172/JCl123159. be cytotoxic to Schwann cells through the formation of inclusion bodies and vacuoles, whereas proliferation of Langerhans cells in the skin is a by-product of inflammation and leads to the loss of intraepidermal nerve fibers. Similarly, inflammatory mechanisms can induce the accumulation of macrophages in dorsal root ganglia (DRG), leading to injury and peripheral neuropathy (7-9).

Cisplatin, a platinum-based antineoplastic drug, is one of the most widely prescribed chemotherapeutic agents. Cisplatin exerts its antineoplastic effects through the formation of platination products in nuclear DNA (10-12), with the most common product being a guanine-guanine intrastrand cross-link. If these DNAbase cross-links are not repaired, they disturb DNA's helical conformation and interrupt replication and transcription. The stalling of replication forks and/or RNA polymerases eventually induces signaling pathways leading to cell cycle arrest or cell death $(5,10-$ 12). Although this is a desired outcome in cancer cells, we would like to avoid it in healthy cells.

Nucleotide excision repair (NER) is the primary cellular mechanism for repairing cisplatin-induced DNA intrastrand cross-links (13-16). NER requires the cooperation of major protein groups in mammalian cells (16) and can be divided into 2 subpathways: global-genome NER (GG-NER) and transcription-coupled NER (TC-NER). TC-NER and GG-NER use different protein complexes in the initial recognition of DNA damage. GG-NER, which prevents mutagenesis and genomic instability, requires xeroderma pigmentosum group C, specifically XPC-RAD23B, and DNA damage-binding complexes to survey the genome and recognize helix-distorting DNA cross-links. TC-NER initiates when RNA polymerase stalls at DNA lesions, signaling Cockayne syndrome (CS) proteins CSA and CSB to bind DNA damage, remove transcription-blocking lesions, and restore transcription. Defects in TC-NER cause multiple genetic dis- 

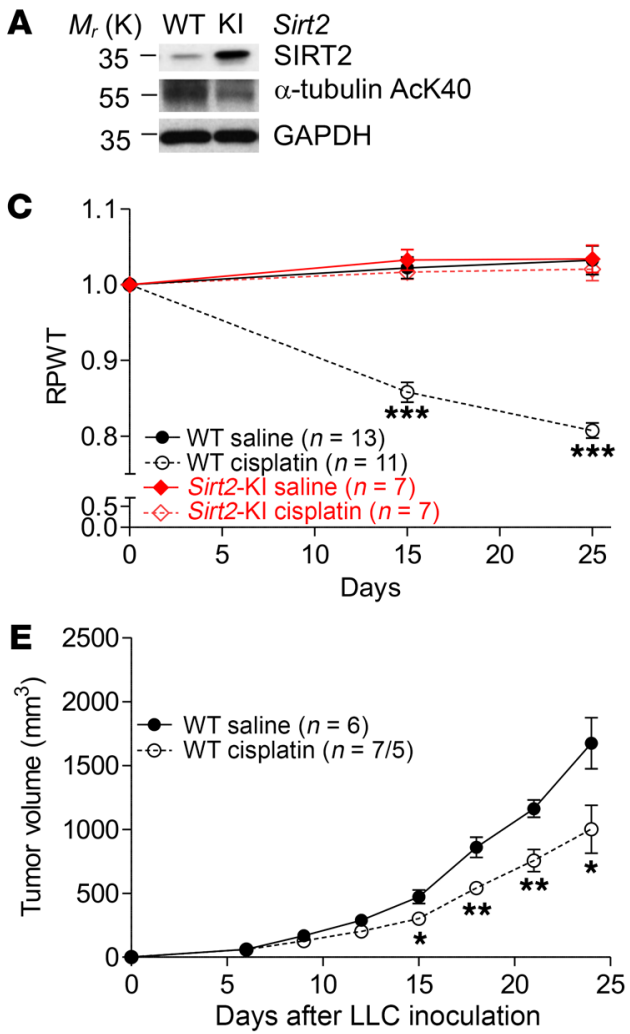

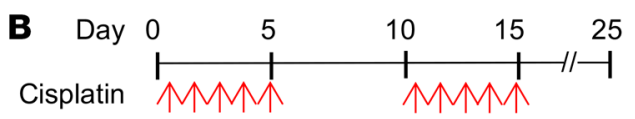

D
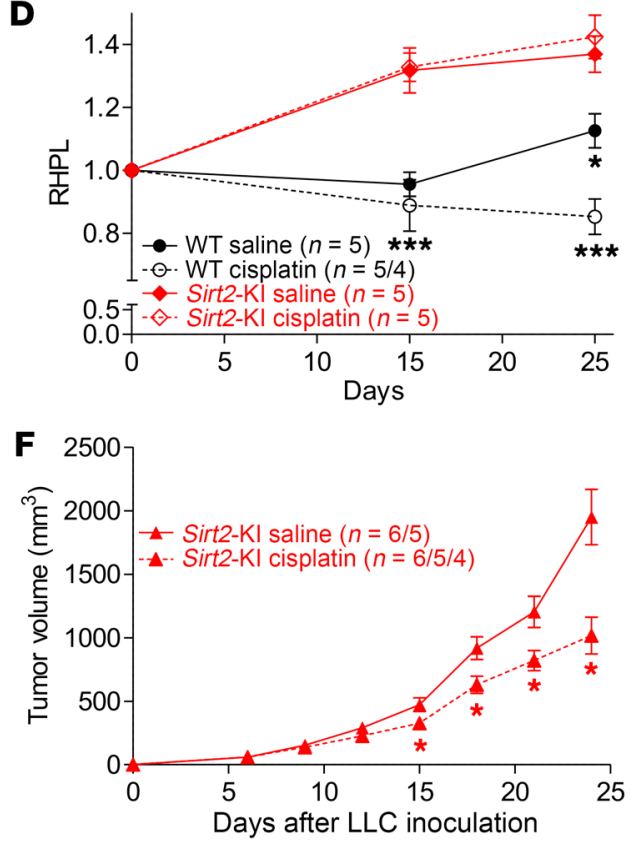

Figure 1. SIRT2 protects mice from cisplatin-induced peripheral neuropathy (CIPN). (A) Western blots show that SIRT2 expression and deacetylase activity increased, as indicated by decreased levels of $\alpha$-tubulin K40 acetylation (AcK40), in Sirt2-knockin (Sirt2-KI) mice, as compared with WT C57BL/6 mice $(n=3)$. (B) Treatment regimen to induce CIPN. (C) Mechanical allodynia measured by von Frey tests and (D) thermal algesia measured by hot plate tests in KI and WT mice before and after cisplatin treatment. RPWT, relative paw-withdrawal threshold, and RHPL, relative hot plate latency, are the pressure and time values in cisplatin- and saline-treated mice normalized to their respective baseline values. (E) C57BL/6 (Sirt2-WT) and (F) Sirt2-KI mice bearing LLC tumors were treated with 2 cycles of cisplatin or saline. Tumor growth was monitored for 25 days. Varying sample sizes represent groups in which mice reached early exit criteria with maximal tumor burden $\left(2,000 \mathrm{~mm}^{3}\right)$ or died of cisplatin toxicity. Statistical significance was assessed using 2-way ANOVA with Bonferroni's correction (C and D) or 2-tailed Student's $t$ test with Welch's correction $(\mathbf{E}) .{ }^{*} P<0.05 ;{ }^{* *} P<0.01 ;{ }^{* * *} P<0.001$.

orders whose symptoms include photosensitivity; intellectual, developmental, and physical disability; and the progeria-like features of CS. XPA, a central coordinator of both GG-NER and TC-NER, binds to altered nucleotides in ssDNA and facilitates DNA damage verification by the TFIIH complex, launching the NER process (15, 17-20).

It is well documented that hypersensitivity to mechanical and thermal stimuli is commonly observed after preferential damage to DRG sensory fibers (21-23). The DRG could have a particularly important role in CIPN. This may be due, in part, to the lack of the blood-brain barrier in the peripheral nervous system and the consequent exposure of its neurons to endogenous and exogenous agents, such as metabolites, inflammatory molecules, and environmental contaminants. Previous studies demonstrated that cisplatin preferentially binds to DRG neurons' DNA with a high propensity for platinum adduct formation $(12,24,25)$. Therefore, it is a plausible hypothesis that the efficiency of TC-NER-mediated repair of cisplatin-induced DNA cross-links might play a critical role in protecting postmitotic neurons from cytotoxicity and CIPN.

SIRT2 is an $\mathrm{NAD}^{+}$-dependent deacetylase that plays a role in multiple biological processes, such as longevity, lipid and glucose homeostasis, tumor suppression, and neurodegenerative disorders (26-30). Recent studies revealed the protective action of
SIRT2 against tissue injury from oxidative stress (31-33). SIRT2 is a cytoplasmic/nuclear protein $(34,35)$, and its expression level and subcellular localization are regulated through stimuli such as diet, oxidative stress $(33,34)$, and cell cycle progression $(36,37)$. It is reported that activation of SIRT2 by resveratrol $(38,39)$ and nicotinamide riboside, a naturally occurring vitamin precursor of $\mathrm{NAD}^{+}$ (40), alleviates diabetic neuropathic pain in animal studies $(41,42)$.

The current study examined the effect of SIRT2 expression on cisplatin-induced DRG neuron injury and CIPN in mouse models. We report that SIRT2 reduces neuronal cell death after cisplatin treatment and protects mice against CIPN. We discovered what we believe to be a novel function of SIRT2, but not SIRT1, in promoting TC-NER to repair cisplatin-induced DNA cross-links, thereby protecting neurons from cisplatin-mediated cytotoxicity.

\section{Results}

SIRT2 protects mice from CIPN. To determine if SIRT2 plays a role in CIPN, we examined CIPN using genetic murine models in which SIRT2 was expressed normally in wild-type (WT) mice and overexpressed in transgenic Sirt2-knockin mice (Sirt2-KI) that contain 3 copies of the Sirt2 gene (36). The expression and deacetylase activity of SIRT2 in WT and Sirt2-KI mice were confirmed, via Western 

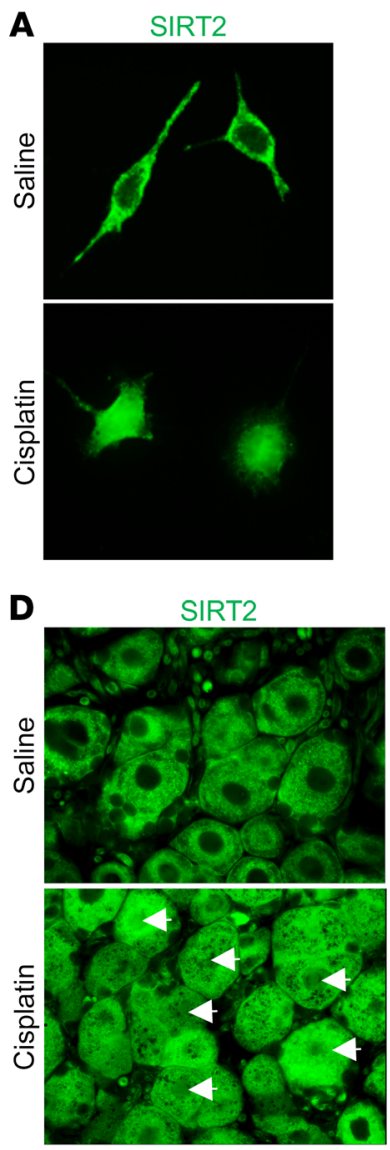

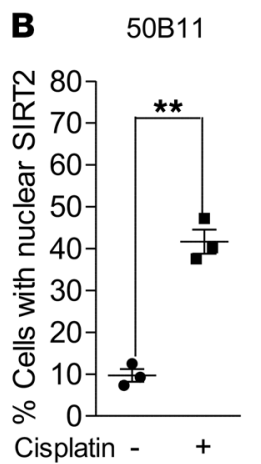

$\mathbf{E}$

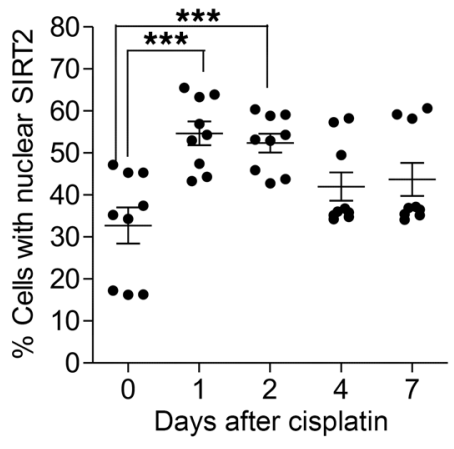

C

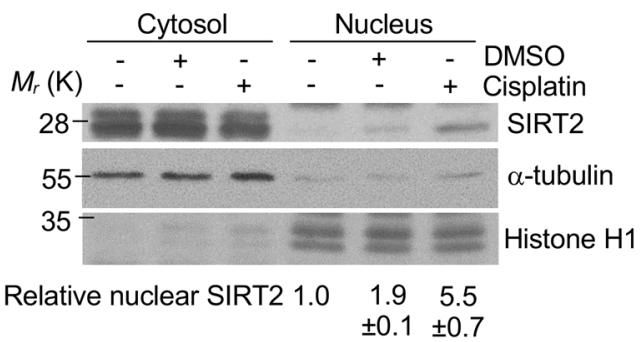

Figure 2. Cisplatin induces SIRT2 nuclear accumulation. (A) Representative images (original magnification, $\times 400$ ) of immunofluorescence-stained SIRT2 and (B) quantification of nuclear SIRT2 in neuronally differentiated 50B11 cells treated with saline or 16 hours of cisplatin $(n=3)$. (C) Nuclear and cytoplasmic fractionated Western blot demonstrating that cisplatin increased nuclear accumulation of SIRT2 in 50B11 cells $(n=2)$. Histone $\mathrm{H} 1$ and $\alpha$-tubulin served as the nuclear and cytoplasmic protein loading controls, respectively. Relative nuclear SIRT2 represents band intensity of nuclear SIRT2 normalized to the corresponding histone $\mathrm{H} 1$ relative to nuclear SIRT2 intensity without treatment. (D) Representative images $(\times 630)$ of immunofluorescence-stained paraffin sections of DRGs from saline-treated and cisplatin-treated C57BL/6 mice 48 hours after treatment. An anti-SIRT2 antibody was used to visualize SIRT2, and arrows indicate neurons with nuclear SIRT2. (E) Dynamic distribution of neurons with nuclear SIRT2 during a 7-day period after cisplatin $(n=9)$. Images represent 1 of 3 replicates. Statistical significance was assessed using a 2-tailed Student's $t$ test with Welch's correction (B) or 1-way ANOVA with Dunnett's correction (E). ${ }^{* *} P<0.01 ;{ }^{* *} P<0.001$ blot, by measuring the levels of SIRT2 protein and of acetylation of K40 (AcK40) on $\alpha$-tubulin, a classic SIRT2 substrate (Figure 1A). CIPN was induced in C57BL/6 adult mice with a clinically relevant dose of cisplatin $(2.3 \mathrm{mg} / \mathrm{kg}$; refs. $43-45)$. Because CIPN commonly manifests in patients as hyperalgesia, an increased perception of pain $(2,5,6)$, we assessed mechanical thresholds (i.e., paw-withdrawal pressure in grams) with von Frey filaments and thermal thresholds (i.e., paw-withdrawal latency in seconds) with dynamic hot plates. Reported literature on the effect of cisplatin on thermal sensitivity currently shows a discrepancy in murine models. Although one study saw no difference in thermal hyperalgesia on hot plate studies (46), others demonstrate enhanced sensitivity to noxious heat following cisplatin, more so than oxaliplatin, treatment $(43,47,48)$. Likewise, mechanical hyperalgesia has been documented in mice as measured by decreased pressure tolerance on von Frey filament tests. As depicted in Figure 1B, we began the first cycle of cisplatin 24 hours after the baseline mechanical and thermal thresholds were measured. Daily intraperitoneal (i.p.) cisplatin injections were given on days 1 to 5 , followed by 5 days of rest. The second cycle of cisplatin included daily i.p. injections on days 11 to 15 , and mechanical and thermal thresholds were tested on days 0,15 , and 25 . WT mice showed relatively stable mechanical and thermal thresholds when given saline injections during the treatment course. However, after cisplatin treatment, WT mice exhibited significant decreases in both relative mechanical and thermal thresholds on days 15 and 25 (Figure 1, C and D). Intriguingly, Sirt2-KI mice displayed a significantly higher resistance to
CIPN after both cycles of cisplatin injections, maintaining the mechanical threshold and gaining thermal tolerance compared with baseline measurements (Figure 1, C and D). Both mechanical and thermal thresholds showed similar trends in male and female WT mice at baseline and after cisplatin treatment (Supplemental Figure 1, A and C; supplemental material available online with this article; https://doi.org/10.1172/JCI123159DS1). There was no significant difference observed between females and males in Sirt2KI mice (Supplemental Figure 1B).

To more closely mimic cancer patients in clinical conditions, we further examined whether the presence of tumors influences the protective effect of SIRT2 on CIPN. WT and Sirt2-KI mice bearing xenograft tumors from Lewis lung carcinomas (LLCs) were treated with cisplatin, and we observed the same protective effect of high SIRT2 expression against CIPN in Sirt2-KI mice (data not shown). To examine if SIRT2 expression levels in mouse influence cisplatin-mediated tumor control, tumor volume was measured in WT and Sirt2-KI mice in the presence and absence of cisplatin. Interestingly, despite the significant protective effect of SIRT2 against CIPN, SIRT2 expression did not affect LLC response to cisplatin in either WT or Sirt2-KI mice (Figure 1, E and F). Both WT and Sirt2-KI mice showed significantly reduced tumor volume upon receipt of cisplatin, compared with mice not treated with cisplatin. Together, these results strongly suggest that overexpression of SIRT2 protects against peripheral neuropathy manifested as mechanical and thermal allodynia (i.e., sensitization) without hindering tumor suppression caused by cisplatin treatments. 



Figure 3. SIRT2 regulates TC-NER-mediated DNA cross-link repair. TC-NER efficiency was analyzed using a TC-NER reporter assay. Luciferase expression and activity from a cisplatin-induced cross-linked CMV-firefly luciferase plasmid was calculated as a percentage of the expression/activity from a CMV-firefly luciferase control plasmid. (A) XPA-mutated cells were defective in NER-mediated repair of the cross-linked luciferase plasmid compared with XPA-complemented (C-XPA) cells $(n=5)$. (B) NER-mediated repair of cross-links was diminished in Sirt2-KO neuronally differentiated $50 B 11$ and PC12 cells. Reexpression of WT-SIRT2, but not the enzymatically inactive mutant, HY-SIRT2, in KO cells restored NER efficiency $(n=3)$. Western blots (left inset) show rat SIRT2 (rSIRT2) and human SIRT2 (hSIRT2) expression in these cells. Vinculin was the protein loading control. (C) Dot blot for level of DNA-platinum adduct measurement in neuronally differentiated 50B11 cells following cisplatin or saline administration $(n=3)$. (D) Quantification of DNA dot blot $(n=2)$. Sirt2 KO resulted in an increase in DNA-platinum adduct formation following cisplatin treatment. Reexpression of WT-SIRT2 restored DNAplatinum adduct repair. Statistical significance was assessed using 2-tailed Student's $t$ test with Welch's correction (A), 1-way ANOVA with Dunnett's correction (B), or 2-way ANOVA with Bonferroni's correction (D). ${ }^{* *} P<0.01 ;{ }^{* *} P<0.001$.

Cisplatin induces SIRT2 nuclear accumulation in DRG sensory neurons. To explore the mechanisms involved in SIRT2-mediated protection against CIPN, we examined whether SIRT2 expression or localization in peripheral neurons is altered by cisplatin treatment. Although Schwann cells, macrophages, and Langerhans cells are possible targets of CIPN, we focused on DRG sensory neurons in this study. We measured SIRT2 expression and subcellular location within neuronally differentiated 50B11 cells, immortalized neuronal cells derived from rat DRG sensory neurons, with immunofluorescence (IF) staining and fractionated Western blotting after saline or cisplatin treatments. Differentiation and axonal elongation is easily induced in culture with the growth factor forskolin, and the majority of cells stop dividing and begin to extend long neurites within hours (49). All data acquisition in this study was performed after neuronal differentiation of the 50B11 and PC12 cells, a commonly used cell model for differentiated peripheral neurons.

We observed a moderate increase in SIRT2 expression (approximately 1.5-fold) after cisplatin exposure (data not shown). Interestingly, we observed cisplatin-induced nuclear accumulation of SIRT2 that resulted in an approximately 4 -fold increase in nuclear SIRT2 in 50B11 neurons, as assessed via IF (Figure 2, A and B). A 5.5-fold increase was observed when assessed via frac- tionated Western blotting (Figure 2C). Similar SIRT2 expression and nuclear accumulation responses were observed after cisplatin treatment in PC12 cells (Supplemental Figure 2, A-C).

To validate these findings in vivo, we examined cisplatininduced SIRT2 localization in DRG sensory neurons from WT mice. IF staining of DRG tissue revealed that cisplatin induced a significant increase in SIRT2 nuclear accumulation that peaked on day 1 after cisplatin treatment (approximately 55\% of DRG neurons from cisplatin-treated mice had nuclear SIRT2 versus $32 \%$ before cisplatin treatment) and returned to basal levels on day 4 after cisplatin treatment (Figure 2, D and E). Consistent with the in vitro cultured neurons, SIRT2 expression in DRG neurons moderately increased after cisplatin treatment (data not shown).

SIRT2 promotes NER in neuronally differentiated cells. To investigate the underlying molecular mechanism by which increased SIRT2 expression protects mice from CIPN, we examined if SIRT2 is involved in NER to remove cisplatin-induced DNA cross-links in DRG neurons. We measured TC-NER by employing a dual-luciferase reporter assay in which a cisplatincross-linked CMV-firefly luciferase plasmid (pCMV-Luc XL) or a non-cross-linked CMV-firefly luciferase plasmid (pCMV-Luc) was mixed with TK-Renilla luciferase plasmid (TK-Luc) and 
A
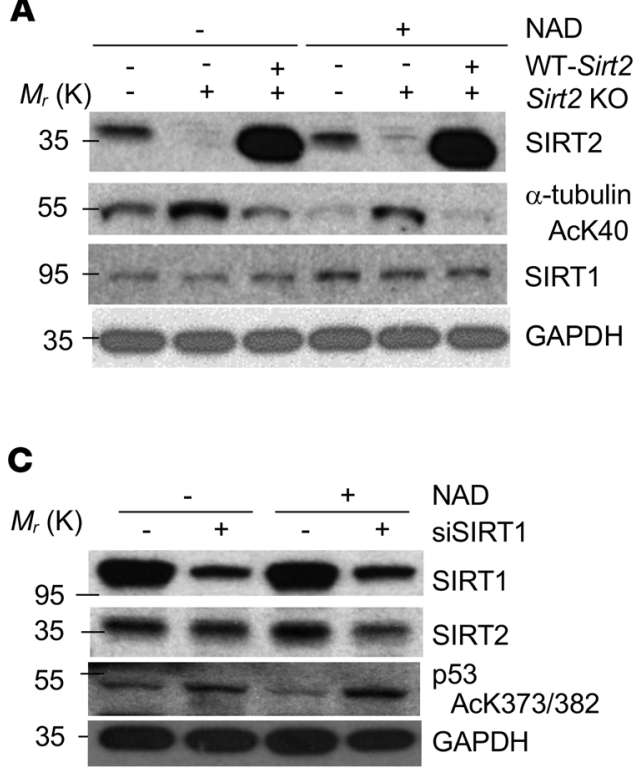
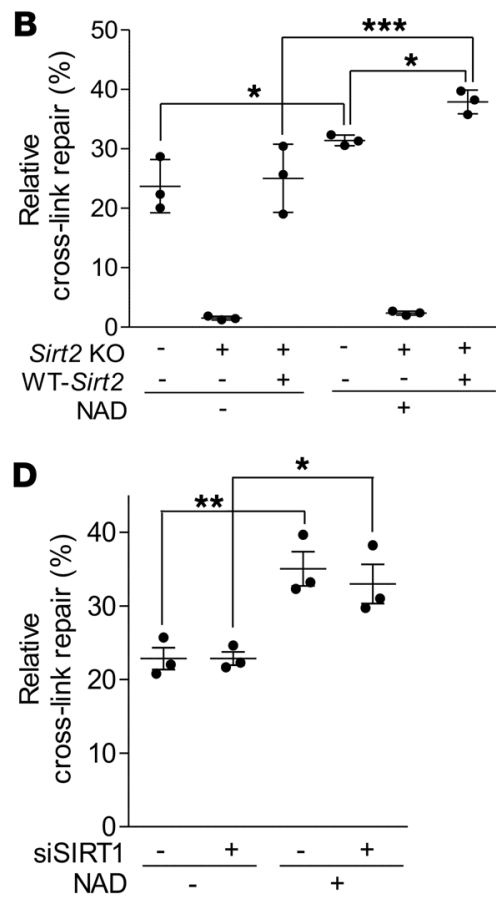

Figure 4. $\mathrm{NAD}^{+}$-mediated enhancement of NER efficiency requires SIRT2 and is independent of SIRT1. (A) Western blot showing the activation effects of $5 \mathrm{nM} \mathrm{NAD}{ }^{+}$on SIRT2 expression and deacetylase activity $(n=3)$, which is indicated by the level of $\alpha$-tubulin AcK40. (B) NAD+ administration enhanced NER efficiencies in a SIRT2-dependent manner in neuronally differentiated $50 \mathrm{~B} 11$ cells $(n=3)$. (C) Western blot showing the effects of administration of $5 \mathrm{nM} \mathrm{NAD}{ }^{+}$on SIRT1 expression and deacetylase activity in 50B11 cells in which SIRT1 expression is modified using siRNA against $\operatorname{SIRT1}(n=3)$. Deacetylase activity is indicated by acetylation of $\mathrm{p} 53$ at K373/K382 (AcK373.382). (D) SIRT1 expression and activity did not affect $N A D^{+}$-mediated enhancement of NER efficiencies in $50 B 11$ cells $(n=3)$. Statistical significance was assessed using 2-way ANOVA with Bonferroni's correction (B and $\mathbf{D}$ ). ${ }^{*} P<0.05$; ${ }^{*} P<0.01$; ${ }^{* *} P<0.001$ cotransfected into cultured cells. All plasmids are nonreplicable in mammalian cells and are not susceptible to silencing via methylation. Firefly and Renilla luciferase activities, which are dependent on their levels of transcription, were measured 24 hours after transfection. CMV-Luc activity was normalized to TK-Luc activity, and the ratio of pCMV-Luc XL activity (indicative of repair of the cross-links) to CMV-Luc activity per sample was calculated as TC-NER efficiency (i.e., percentage of relative cross-link repair) (50). An isogenic pair of XPA-deficient (XPA) and XPA-complemented (C-XPA) fibroblast cells was included as negative and positive controls, respectively, for the TC-NER reporter assay. Consistent with previous reports (5052), TC-NER dysfunction in XPA cells was evident by its inability to repair the cross-linked luciferase plasmid (Figure $3 \mathrm{~A}$ ). In $\mathrm{C}$-XPA cells, complemented XPA engaged in NER, with repair efficiency reaching $50 \%$, nearly a 50 -fold increase in NER efficiency compared with that in XPA cells (Figure 3A). As shown in Figure 3B, neuronally differentiated 50B11 cells exhibited a $27 \%$ NER efficiency. Knockout (KO) of Sirt2 in 50B11 cells with the CRISPR/Cas9 lentivirus system $(53,54)$ resulted in abolishing NER, and repair efficiency dropped to $6 \%$. Reintroduction of WT Sirt2 restored NER repair efficiency to $36 \%$, but NER was not restored with the reintroduction of the catalytically inactive mutant HY-Sirt2 (Figure 3B), confirming that abolished NER is the result of Sirt2 KO instead of an off-target effect. The significantly higher NER efficiency after reconstitution of Sirt2, compared with that in parental cells, was consistent with the higher expression levels of exogenous SIRT2 than endogenous SIRT2, as seen on the Western blot (Figure 3B). A similar SIRT2 effect on NER was observed in PC12 cells, as shown in Figure 3B.

To confirm that SIRT2-mediated promotion of NER translates to an increase in the repair of cisplatin-DNA adducts in neuronally differentiated cells, we performed dot blot assays using a mono- clonal antibody specific for cisplatin-GG adducts, a major mechanism of cisplatin-induced DNA damage (8-10). Compared with parental DRG neurons, increased amounts of DNA adduct were detected in Sirt2-KO cells (Figure 3, C and D). Reconstitution of WT Sirt 2 expression resulted in reduction of DNA adducts. These data support the role of SIRT2 in promoting NER-mediated repair of cisplatin-induced DNA adducts in neurons.

TC-NER efficiency is specifically dependent on SIRT2, but not SIRT1, in neuronally differentiated cells. SIRT1 is another member of the sirtuin family that shares certain biochemical functions with SIRT2 and has been reported to be involved in DNA repair (55-57). Once we established that SIRT2 protects mice against CIPN through promoting TC-NER of cisplatin-induced DNA cross-links in immortalized neuronal cells, we investigated whether SIRT1 is also involved in regulating TC-NER. Both SIRT1 and SIRT2 are NAD ${ }^{+}$-dependent deacetylases and are known to increase their activity in response to elevated $\mathrm{NAD}^{+}$levels. These levels can be increased by directly supplementing cell culture media with $\operatorname{NAD}^{+}(41,42,58)$.

We found that supplementing $50 \mathrm{~B} 11$ cells with $\mathrm{NAD}^{+}$alone activates SIRT2 deacetylase activity, as evidenced by a decreased level of acetylated $\alpha$-tubulin (Figure 4A). Activation of SIRT2's deacetylase activity was associated with a moderate but significant increase in NER efficiency, as compared with a control cell culture that was not supplemented with $\mathrm{NAD}^{+}$(Figure $4 \mathrm{~B}$ ). Importantly, $\mathrm{NAD}^{+}$supplementation did not enhance NER efficiency in 50B11 Sirt2-KO cells, but the effect of $\mathrm{NAD}^{+}$supplementation on NER was restored once Sirt 2 was reconstituted in the 50B 11 Sirt2-KO cells (Figure 4B).

To determine if SIRT1 also plays a role in $\mathrm{NAD}^{+}$-mediated NER enhancement, we examined NER efficiency after supplementing $\mathrm{NAD}^{+}$in $50 \mathrm{~B} 11$ cells with or without SIRT1 inhibition using siRNA. The siRNA-mediated SIRT1 knockdown was verified by the inhibition of SIRT1 expression and the suppression of SIRT1 deacetylase activity, evidenced by increased acetylation of p53, a well-defined 
A

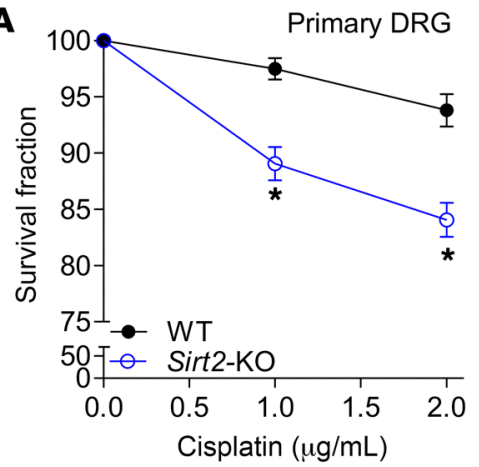

B

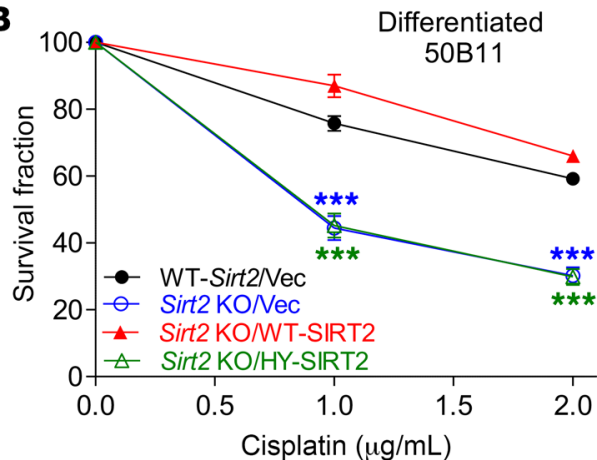

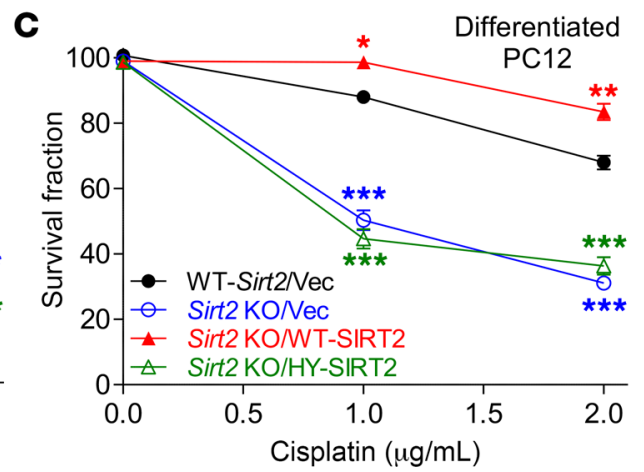
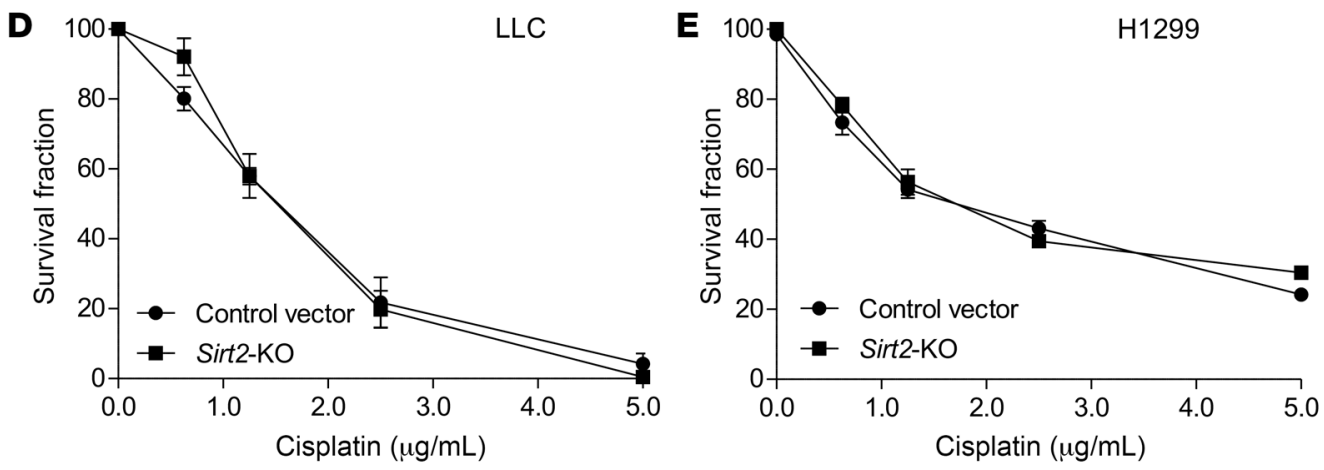

Figure 5. SIRT2 protects neuronal cells, but not tumor cells, from cisplatin-induced cytotoxicity. (A) Sirt2 KO in primary DRG neurons resulted in decreased cell survival after cisplatin treatment. Data were analyzed by 2-tailed Student's $t$ test $(n=3)$. (B and C) The viability of neuronally differentiated 50B11 (B) and PC12 (C) cells after various doses of cisplatin treatment was measured with trypan blue staining. Reexpression of WT-SIRT2, but not the enzymatically inactive mutant, HY-SIRT2, in Sirt2-KO 50B11 and PC12 cells increased cell resistance to cisplatin cytotoxicity. One-way ANOVA demonstrated a main effect for cell genotype $(P<0.001)$. ${ }^{*} P<0.05$; ${ }^{* *} P<0.01$; ${ }^{* *} P<0.001$ denote significance levels detected among various cell genotypes and at different time points by Tukey's post hoc analysis. (D and E) The viability of control vector and Sirt2-KO LLC (D) and H1299 (E) cells after varying doses of cisplatin treatment, as measured by trypan blue staining. Sirt2 KO in LLC and H1299 cells shows no difference in cell viability following cisplatin treatment, as analyzed by 2-tailed Student's $t$ test.

substrate for SIRT1 deacetylase activity (Figure 4C). As shown in Figure 4D, siRNA-mediated SIRT1 inhibition did not affect NER in $50 \mathrm{~B} 11$ cells. However, $\mathrm{NAD}^{+}$supplementation significantly enhanced NER efficiency, independently of SIRT1 activity. These results further support SIRT2-specificity of protection against CIPN and the requirement for SIRT2's function in TC-NER.

Together, these in vitro results strongly suggest that NERmediated DNA repair of differentiated neuronal cells is specifically dependent on SIRT2 and can be enhanced by activation of SIRT2 with $\mathrm{NAD}^{+}$supplementation.

SIRT2 protects neurons from cisplatin-induced cytotoxicity. TC-NER is the key defense mechanism against cisplatin-induced cytotoxicity in differentiated neuronal cells. To further substantiate the role of SIRT2-dependent NER in cisplatin-induced neuron injury, we investigated the protective effects of SIRT2 in DRG neurons that were damaged from cisplatin-induced cytotoxicity. Cell death was measured by positive trypan blue staining in primary DRG neurons, from either WT or Sirt2-KO mice, and neuronally differentiated 50B11 and PC12 cells in which SIRT2 expression was manipulated using the $\mathrm{KO}$ and restoration approaches detailed above. Sirt $2 \mathrm{KO}$ caused a significant decrease in survival following cisplatin treatment (Figure 5, A-C, and Supplemental Figure 3, A-C). The decreased survival in 50B11 and PC12 cells was rescued by reexpression of WT-SIRT2 in comparison with vector rescue controls (Figure 5, B and C, and Supplemental Figure 3 , B and C). Interestingly, higher expression levels of exogenous SIRT2 in rescued cells (Supplemental Figure 3, B and C) resulted in improved cell survival compared with parental 50B11 and PC12 cells with endogenous SIRT2 expression plus vector control (Figure 5, B and C). We further found that expression of the enzymatically deficient HY-SIRT2 mutant did not restore cells' resistance to cisplatin-induced cytotoxicity (Figure 5, B and C), indicating that the deacetylase activity of SIRT2 is not only critical for NER function (Figure 4B) but is also a requisite factor for the resistance of 50B11 and PC12 cells against cisplatin-induced neuronal death. Importantly, SIRT2 expression had no effect on lung cancer cell cytotoxic response to cisplatin treatment (Figure 5, D and E, and Supplemental Figure 3, D and E).

SIRT2-mediated protection against CIPN depends on its function in NER. To further validate the role of NER in SIRT2-mediated protection against CIPN, we studied the effects of in vivo NER inhibition using spironolactone (SP), a small-molecule NER inhibitor. SP promotes the degradation of XPB, a transcription factor subunit vital for NER function (59). The efficacy of SP in XPB degradation and its effect on NER function in neuronally differentiated 50B11 cells were evaluated via Western blotting (Figure 6A) and the dual-luciferase reporter assay (Figure 6B). SP effectively inhibited XPB expression, resulting in an approximately 5.4-fold 
A

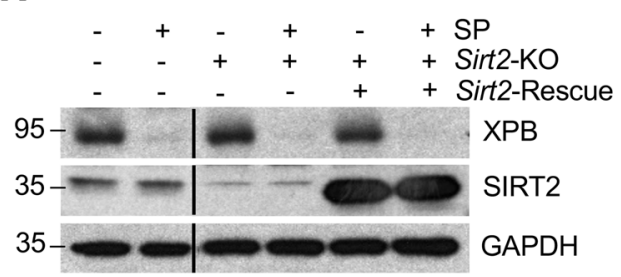

B

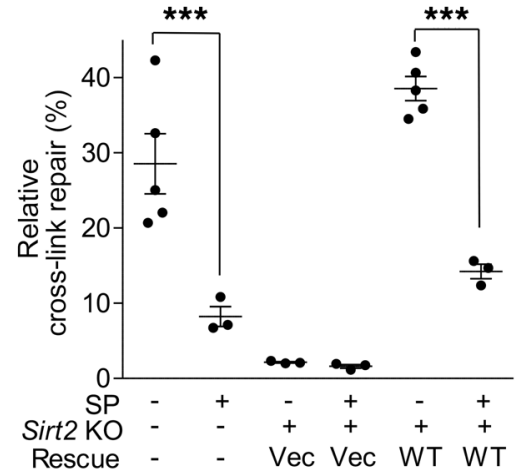

c

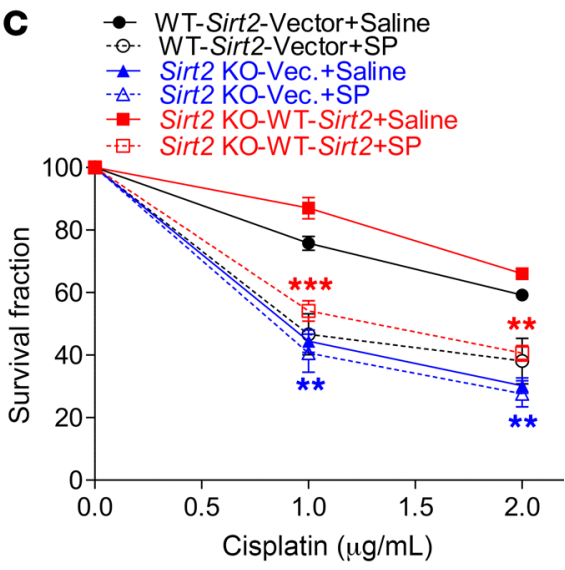

Figure 6. SIRT2 protection of neuronally differentiated cells from cisplatin cytotoxicity is dependent on NER function. (A) Western blot showing XPB and SIRT2 expression in neuronally differentiated 50B11 cells with varying Sirt2 gene modifications with or without spironolactone (SP) treatment. The lanes were from the same gel but were not contiguous. Blots represent 1 of 3 replicates. (B) NER efficiency was significantly decreased by Sirt2 KO or treatment of $50 B 11$ cells with $10 \mu \mathrm{M} \mathrm{SP}$, which can increase the degradation of the key NER protein, XPB, and thereby inhibit NER. Sirt2 KO led to significantly lower NER than SP. Reexpression of WT-SIRT2 rescued NER only in the absence of SP-mediated interruption of NER function. $n=5$ or 3 . (C) SP pretreatment increased cisplatin-induced cytotoxicity in 50B11 cells. SP had no further effect on cisplatin-induced cell killing in Sirt2-KO and NER-deficient 50B11 cells. WT and Sirt2-KO cells were rescued with WT-SIRT2 to rule out off-target effects and showed no difference in survival. The viability of $50 B 11$ cells after cisplatin treatment at various doses was measured with trypan blue staining. Statistical significance was assessed using 2-way ANOVA with Bonferroni's correction (B and $\mathbf{C})$. ${ }^{* *} P<0.01 ;{ }^{* *} P<0.001$.

reduction in NER efficiency (6.9\%) compared with control 50B11 cells (37.5\%). Sirt2 KO alone in 50B11 cells lowered NER to $2.0 \%$, which was significantly lower than SP treatment alone. SP inhibition of NER in Sirt2-KO 50B11 cells did not further decrease the repair efficiency. Upon reintroduction of Sirt2, repair efficiency was restored to $39.6 \%$, similar to parental 50B11 cells, and was reduced to $13.5 \%$ upon addition of SP (Figure 6B).

In addition to its inhibition of SIRT2-mediated NER, SP pretreatment of SIRT2-reconstituted Sirt2-KO 50 OB11 cells significantly increased cisplatin-induced neuronal cell death compared with saline-treated controls (Figure 6C). However, SP pretreatment had no effect on sensitivity to cisplatin-induced cell death in empty vector-expressing Sirt2-KO 50B11 cells, which are defective in NER (Figure 6C). SP alone was not toxic and had no effect on viability of neuronally differentiated parental 50B11 or Sirt2KO 50B11 cells with or without SIRT2 reconstitution (Supplemental Figure 4). Together, our data suggest that SIRT2-mediated protection of neurons from cisplatin-induced cytotoxicity requires its function in TC-NER.

We reasoned that if the CIPN protection observed in Sirt2-KI mice was dependent on SIRT2 function in promoting NER in DRG neurons, pharmacological NER inhibition with SP would render DRG neurons susceptible to CIPN in Sirt2-KI mice, but have no effect in Sirt2-KO mice. Having confirmed the effectiveness of SP on NER inhibition in 50B11 cells (Figure 6B), cisplatin, alone and in combination with SP, was administered to Sirt2-KI and Sirt2-KO mice via daily i.p. injections. Control groups of both mouse types were administered saline. The injections were given on days 1 to 5 and 11 to 15 , and mechanical and thermal thresholds were measured on days 15 and 25 (Figure 7A). Once again, Sirt2-KI mice that received cisplatin injections resisted CIPN, i.e., there were no significant changes in mechanical and thermal thresholds among Sirt2-KI mice that received cisplatin alone on days 15 and 25 (Fig- ure 7, B and C). However, a significant reduction in mechanical (13\% on day 15 and $15 \%$ on day 25$)$ and thermal thresholds (18\% on day 15 and 23\% on day 25) was seen in Sirt2-KI mice after they received a combination of SP and cisplatin (Figure 7, B and C). In contrast to Sirt2-KI mice, Sirt2-KO mice were sensitive to cisplatin alone and showed a significant decrease in both mechanical $(10 \%$ on day 15 and $16 \%$ on day 25$)$ and thermal thresholds (16\% on days 15 and 25) (Figure 7, D and E). Although SP has previously been implicated in decreased nociceptive thresholds, our results found SP treatment alone did not affect the susceptibility of Sirt2-KO mice to CIPN, and we did not observe a difference in mechanical and thermal threshold changes between mice that received cisplatin alone or a combination of SP and cisplatin (Figure 7, D and E). Together, the data suggest a dependence on SIRT2 for intact NER function and CIPN protection.

\section{Discussion}

Peripheral neuropathy, a common side effect of platinum-based anticancer drugs like cisplatin, can considerably impact cancer treatment strategies and patients' quality of life. With no effective prevention or treatment methods available for patients who suffer from CIPN (1, 3), understanding of its underlying mechanisms is urgently needed. Using genetic mouse models, we demonstrated that SIRT2 overexpression protects mice from CIPN, the first evidence that SIRT2 plays a role in protecting mice from CIPN to our knowledge. Mechanistically, cisplatin induces SIRT2 nuclear accumulation in DRG neurons. We discovered what we believe to be a novel function of SIRT2 to specifically protect DRG sensory neurons from cisplatin-induced cytotoxicity by promoting NER and thereby removing cisplatin-induced DNA cross-links.

We have provided in vivo and in vitro evidence that SIRT2mediated protection against cisplatin-induced neurotoxicity is specifically dependent on its function in promoting TC-NER. 

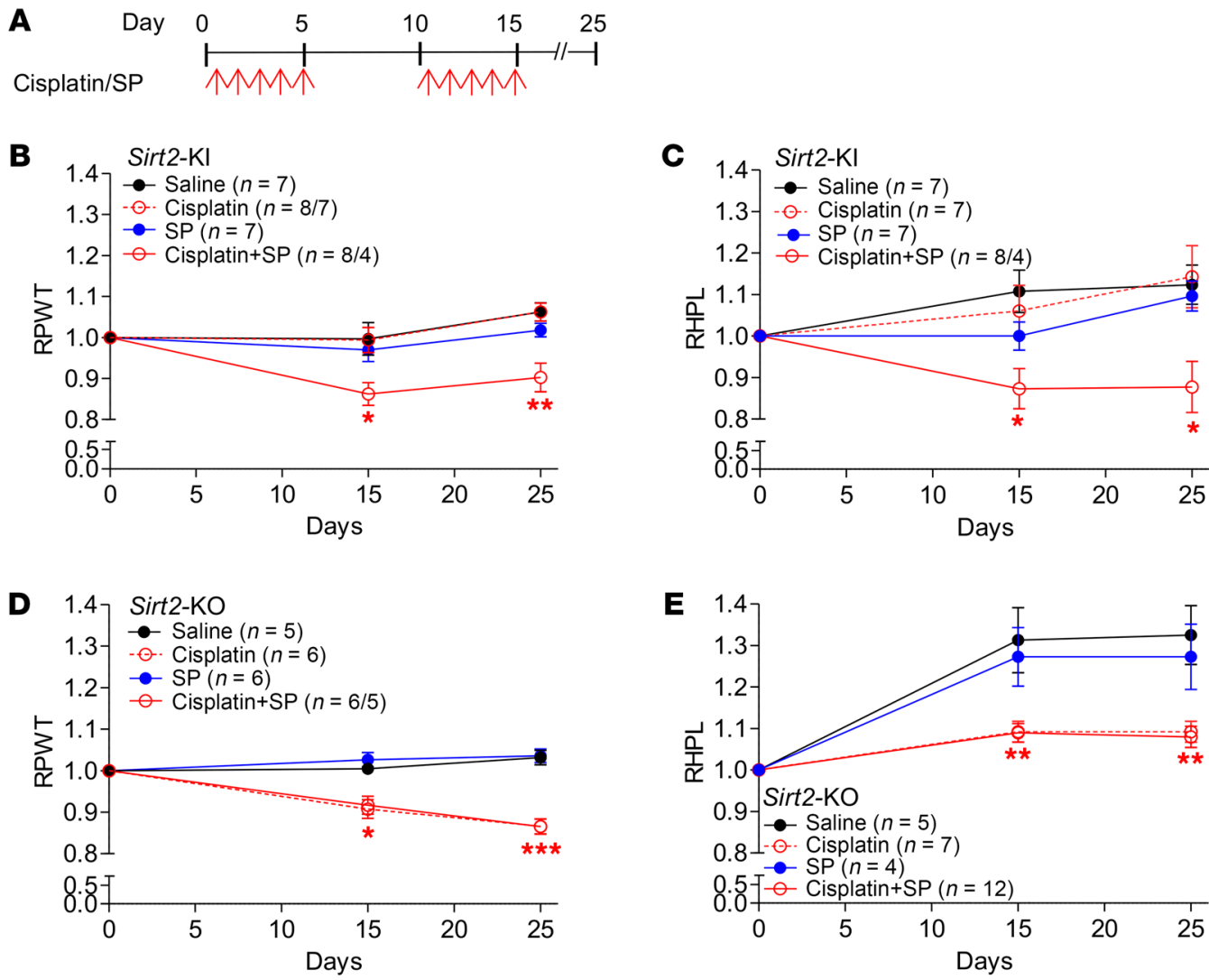

Figure 7. SIRT2's protection of mice from CIPN is dependent on NER function. (A) Cisplatin and spironolactone (SP) treatment regimen for both Sirt2-KI and Sirt2-KO mice. Both strains of mice received the same treatments: saline, cisplatin alone, SP alone, or both cisplatin and SP. Response of (B) Sirt2-KI mice and (D) Sirt2-KO mice to mechanical stimulation, as measured by von Frey tests, is shown as relative paw-withdrawal threshold (RPWT). Response of the same Sirt2-KI mice (C) and Sirt2-KO mice (E) to thermal stimulation, as measured by hot plate tests, is shown as relative hot plate latency (RHPL). Varying sample sizes were due to the death of some mice from treatment toxicity. Statistical significance was assessed using 1-way ANOVA with Dunnett's correction (B-E). ${ }^{*} P<0.05 ;{ }^{* *} P<0.01 ;{ }^{* *} P<0.001$.

Cisplatin-induced accumulation of SIRT2 in the nuclei of DRG neurons may allow SIRT2 to participate in the repair of cisplatin-generated DNA damage (Figure 2). There are 7 sirtuins in mammalian cells, SIRT1-7, and among them, SIRT6 and SIRT7 have been reported to function in DNA repair (60-63); however, whether they are involved in protection against CIPN needs to be further investigated. SIRT1, like SIRT2, has been reported to be involved in DNA repair, but results from this study suggest that NER-mediated repair of cisplatin-induced DNA adducts is independent of SIRT1. On the other hand, other mechanisms, including calcium signaling and homeostasis, oxidative stress, changes in cell signaling cascades, and mitochondrial dysfunction, have been suggested to contribute to cisplatin-induced neurotoxicity (64-69). Other cytotoxic drugs, such as paclitaxel, induce peripheral neuropathy through mechanisms other than DNA damage. Interestingly, nicotinamide riboside, a precursor of $\mathrm{NAD}^{+}$and activator of SIRT1 and SIRT2, has been shown to alleviate paclitaxel-induced peripheral neuropathy independently of DNA damage repair (38). Whether other sirtuins play a role in CIPN, and if SIRT2's protective effect against CIPN is also involved in regulating these proposed mechanisms, is yet to be determined.

Cisplatin-induced neurotoxicity involves DRG neuronal death (21-23). Our study showed that SIRT2-dependent NER is a key factor in determining the sensitivity of DRG neurons to cisplatin-induced cytotoxicity and protecting mice from CIPN. SIRT2's function in promoting NER varies in the cancer cell lines examined (data not shown). In contrast to the protective effect of SIRT2 in terminally differentiated postmitotic DRG neurons, it appears that an association does not exist among SIRT2 expression, NER efficiency, and cisplatin-induced tumor cell killing in vitro, nor in LLC tumor controls in vivo (Figures 1 and 5). The differential response to cisplatin treatment between differentiated functional neurons and proliferating tumor cells raises new questions and points to underlying mechanisms that are yet to be determined.

SIRT2's protective effects against CIPN are dependent on TC-NER; however, other cisplatin-induced DNA repair pathways cannot be excluded. Mismatch repair proteins have been implicated in cisplatin-induced cytotoxicity after binding to DNA-cisplatin adducts and initiating apoptosis. Moreover, mismatch repair deficiency has been shown to give rise to proliferating cancer cell cisplatin resistance, leading to worse oncologic outcomes. How mismatch repair affects differentiated functional neurons' response to cisplatin cytotoxicity is yet to be determined. Although we cannot exclude other DNA repair pathways, our results highlight the significance of TC-NER in cisplatin-induced DNA damage of neuronal cells and warrant future research on this pathway. 
SIRT2 possesses deacetylase activity and regulates multiple signaling pathways through deacetylation of its substrate proteins. A number of proteins have been identified as substrates of SIRT2. For example, SIRT2 deacetylates CDK9, activating its kinase activity in response to DNA replication stress (37). SIRT2 also deacetylates PKM2 and regulates its tetramerization and pyruvate kinase activity, leading to suppression of glycolysis (28). SIRT2 has been reported to deacetylate transcription factor NRF2 and regulate cellular iron homeostasis (70). SIRT2-mediated deacetylation on lysine 669 of BubR1 maintains BubR1 expression levels, which decrease with aging (36). SIRT2 also controls spindle organization and chromosome alignment through regulation of the acetylation status of histone H4K16 and $\alpha$-tubulin $(32,71-73)$. In this study, we demonstrated that the deacetylation activity of SIRT 2 is critical for SIRT2-mediated NER of DNA cross-links, and for the neuronal response to cisplatin cytotoxicity. The key players of TC-NER, including RPA, CSB, XPA, and XPD could be directly regulated by SIRT2 through deacetylation or could be indirectly regulated by expression level by deacetylation of transcription factors. However, it is yet to be determined which proteins are the critical targets of SIRT2 deacetylation to directly or indirectly regulate NER efficiency and protect neurons from cisplatin cytotoxicity.

Treatment with SP allowed us to evaluate whether SIRT2's protection against CIPN was dependent on NER-mediated DNA repair; however, SP has also been shown to alter nociceptive behavior in a mouse model. One particular study (74) examined thermally induced, chemogenic, and visceral pain 1 to 2 hours following SP administration and found treated mice were more sensitive to thermal and chemogenic pain, but more tolerant of visceral pain. However, nociceptive behavior was not recorded beyond 2 hours, so our findings on the response to thermal and mechanical stimuli on day 25 in mice given SP compared with saline provide a long-term perspective.

We acknowledge that CIPN manifested differently in thermal versus mechanical hyperalgesia, as evidenced by earlier development of increased sensitivity to mechanical pain compared with thermal pain following cisplatin treatment (Figure 1). One of the potential explanations is that receptors for mechanical and thermal stimulation might differ in their sensitivity to cisplatin cytotoxicity. If mechanical receptors in the hind paw of the mice are more susceptible to cisplatin than thermal receptors, this might explain the varying results seen in our experiments as well as the literature. Although not specific to CIPN, previous studies have identified C-fiber nociceptors that respond to mechanical, but not thermal, stimulation (75). Moreover, subtypes were identified that respond to different types of mechanical stimuli (vibration, proprioception, low and high thresholds, etc.), thermal stimuli (heat, cold, or both), and a combination of these.

Thermal hyperalgesia caused by cisplatin treatment in murine models remains controversial, as conflicting data have been reported in the literature. With careful observation of mouse behavior, we noticed that, as time progresses, the control mice become more tolerant and show a longer latency period before responding to thermal stimulation. In contrast, treatment with cisplatin in WT, Sirt2-KO, and NER-inhibited Sirt2-KI mice produced a reduction in thermal threshold relative to those treated with saline. Factors contributing to this observed phenomenon might include the tendency for learned behavioral responses or development of hyperkeratosis on the paws of the mice. Previous studies have shown learned behavioral responses to lower reaction times during subsequent exposures to the hot plate $(76,77)$. Furthermore, hyperkeratosis might allow control mice receiving saline to become more tolerant, specifically to thermal stimulation assessed by hot plates, while having no effect on mechanical pain measured by von Frey tests. Despite this divergence from the expected heat tolerance of control mice, SIRT2 overexpression appears to protect mice from increased thermal sensitivity and warrants further study.

In summary, our results identify what we believe to be a novel function of SIRT2 in regulating the TC-NER pathway as a key underlying mechanism of protection against CIPN. These preclinical data provide a mechanistic basis to investigate the role of SIRT2 activation in prevention and treatment of CIPN in cancer patients needing platinum-based cancer treatment.

\section{Methods}

Cell culture. The mouse LLC cell line LL/2 (LLC1), human non-small cell lung cancer cell line H1299, and rat pheochromocytoma cell line PC12 were purchased from ATCC. LLC and PC12 cells were cultured in Dulbecco's Modified Eagle's Medium (DMEM) (Gibco) with 10\% fetal bovine serum (FBS), penicillin $(100 \mu \mathrm{g} / \mathrm{mL})$, and streptomycin $(100 \mu \mathrm{g} / \mathrm{mL})(\mathrm{Gibco})$ and maintained in a $37^{\circ} \mathrm{C}$ incubator with $5 \%(\mathrm{v} / \mathrm{v})$ $\mathrm{CO}_{2}$. H1299 cells were cultured in RPMI- 1640 media (Gibco) with $10 \%$ FBS, penicillin $(100 \mu \mathrm{g} / \mathrm{mL})$, and streptomycin $(100 \mu \mathrm{g} / \mathrm{mL})$ and maintained in a $37^{\circ} \mathrm{C}$ incubator with $5 \%(\mathrm{v} / \mathrm{v}) \mathrm{CO}_{2}$. The rat DRG $50 \mathrm{~B} 11$ cell line was provided by Ahmet Hoke (Johns Hopkins University), and cells were maintained in neurobasal medium with $10 \% \mathrm{FBS}, 0.2 \%$ glucose, $0.5 \mathrm{mM}$ L-glutamine, and 1\% penicillin-streptomycin (Gibco) (49).

Mouse strains. C57BL/6 (Sirt2-WT) mice were purchased from the NCI (Charles River Lab), SIRT2 ${ }^{+/+}$and SIRT2 ${ }^{+-}$C57BL/6 (Sirt2-KI) mice were obtained from David Sinclair (Department of Genetics, Harvard Medical School) (33), and SIRT2 ${ }^{-/-}$C57BL/6 (Sirt2-KO) mice were obtained from Tiago F. Outeiro (Department of Neurodegeneration and Restorative Research, Center for Nanoscale Microscopy and Molecular Physiology of the Brain, University Medical Center Göttingen) (72, 73). The genotypes of all mice were verified by PCR-based genotyping. Six- to 8-week-old male or female mice were used, depending on the availability. Homozygous Sirt2-KI is lethal. Heterozygous Sirt2-KI mice result from the breeding of Sirt2-WT (C57BL/6) and heterozygous Sirt2-KI mice, which give birth to mice with Sirt2-WT and heterozygous Sirt2-KI genotypes (33). For the experiments involving Sirt2-KI mice, their littermate Sirt2-WT mice were used. For all the other experiments, C57BL/6 (Sirt2-WT) mice from NCI were used. All mice were bred and maintained in the Department of Laboratory Animal Medicine (DLAM) at the University of Arkansas for Medical Sciences.

CIPN. Peripheral neuropathy was induced by daily i.p. injections of cisplatin (50 mL vial, Fresenius KABI, suspended in saline [0.9\% sodium chloride injection, Hospira]) at $2.3 \mathrm{mg} / \mathrm{kg}$ for 2 cycles, with 5 consecutive daily injections in each cycle and a 5 -day rest in between the 2 cisplatin treatment cycles. On the final treatment day and 10 days thereafter, electronic von Frey tests and hot plate tests were performed successively to evaluate mechanical allodynia and thermal sensitivity.

Tactile allodynia assay (von Frey test). To assess static mechanical pain hypersensitivity in mice, the thresholds of tactile allodynia were 
measured with an electronic von Frey system (Dynamic Plantar Aesthesiometer, Ugo Basile). The mice were placed in a chamber box with a mesh screen floor, and a single, unbending filament was applied vertically to the mid-plantar region of both hind paws with increasing force (grams) until a paw-withdrawal response was elicited. The force at which this response occurred was electronically recorded and was designated as the paw-withdrawal threshold by the apparatus. These steps were repeated 3 times and the average measurement was calculated and recorded $(78,79)$.

Heat hypersensitivity assay (hot plate test). Heat hypersensitivity was tested using a plantar hot plate analgesia meter, as previously described (IITC Life Science Inc) $(43,45)$. The mice were individually placed on a hot plate that was maintained at a temperature of $51.0^{\circ} \mathrm{C} \pm 0.1^{\circ} \mathrm{C}$. The latency (seconds) to the first sign of hind paw licking or jumping or a jump response to avoid thermal pain was taken as an index of pain threshold and was monitored using an electronic timer. Decreases in withdrawal latency corresponded to increased sensitivity to heat stimuli (10-12). Results were reported as the mean value of 3 readings.

Subcutaneous LLC mouse model. WT, Sirt2-KI, and Sirt2-KO C57BL/ 6 male mice 6 to 8 weeks old were inoculated subcutaneously (s.c.) under anesthesia with $5 \times 10^{5}$ LLC1 cells suspended in $100 \mu \mathrm{L}$ ice-cold phosphate-buffered saline (PBS). Each injection was given between the lower abdomen and left and right hind legs. The day of tumor cell implantation was designated as day 0 and tumor size was measured from day 6 through day 30 .

Effect of NER inhibitor on CIPN. Daily oral NER inhibitor SP (50 $\mathrm{mg} / \mathrm{kg}$, resuspended in PBS, from the University of Arkansas for Medical Sciences pharmacy) alone, or together with cisplatin (i.p. injection), was administered to the mice for 5 days following baseline behavior tests. A second 5-day cycle was given following 5 rest days. For mice treated with cisplatin along with SP, 2 cycles of i.p.-injected cisplatin $(2.3 \mathrm{mg} / \mathrm{kg})$ were given as described in the CIPN model.

$I F$. Fresh mouse DRG samples were immediately fixed with formalin following dissection. Cells cultured on coverslips were fixed by paraformaldehyde. Paraffin-embedded DRG tissues were processed as $4-\mu \mathrm{m}$ sections, then deparaffinized and rehydrated. Samples were pretreated to allow antigen retrieval with Target Retrieval Solution, $\mathrm{pH}$ 6.0 (DAKO). Sections and cell culture coverslips were blocked with 3\% $\mathrm{H}_{2} \mathrm{O}_{2}$, followed by blocking with $2 \%$ goat serum/0.1\% Triton X-100/ PBS (1 hour). Slides were then incubated with a mouse monoclonal primary antibody against SIRT2 (catalog PA5-20487, Thermo Fisher Scientific) and anti-NeuN antibody (catalog ab104224, Abcam). These antibodies were used at a dilution of 1:200, with overnight incubation at $4^{\circ} \mathrm{C}$. Slides were then incubated with a mouse monoclonal primary antibody against SIRT2 (catalog PA5-20487, Thermo Fisher Scientific) at a 1:200 dilution, with overnight incubation at $4^{\circ} \mathrm{C}$. Slides were washed with PBS and incubated with secondary goat anti-mouse Alexa Fluor 594-conjugated antibodies or goat anti-rabbit Alexa Fluor 488-conjugated antibodies (1:1000; catalog A-11032 and catalog A-11034, Molecular Probes, Thermo Fisher Scientific), stained with 4',6-diamidino-2-phenylindole (DAPI) for nuclear visualization for 1 to 2 minutes, and analyzed by fluorescence microscopy (Carl Zeiss). Both cytoplasmic and nuclear SIRT2 immunoreactivity were revealed by the SIRT2 antibody described above. The specificity of immunostaining with SIRT2 antibody was confirmed by the absence of staining when preincubating the antibody with an excess of the peptide immunogen. Negative controls had the primary antibody omitted and replaced by nonimmune normal serum from the same species as the primary antibody. Only peripheral neurons with both SIRT2- and DAPI-positive staining in the nuclei located on the same section were counted as cells with SIRT2 nuclear accumulation. SIRT2 subcellular expression was scored separately by 2 independent researchers who were blinded to cisplatin treatment. SIRT2 expression was measured by ImageJ software (NIH).

SIRT2 KO with CRISPR/Cas9 gene editing. Five pairs of SIRT2 single guide RNAs (sgRNAs) designed by the ATUM CRISPR design tools (http://www.atum.bio/catalog/vectors/grna-design) were prepared for screening. The oligos were designed based on the target site sequence (20 bp) and were flanked on the $3^{\prime}$ end by an NGG PAM sequence. Lenti-CRISPR-v2 (Addgene, 52961) contained 2 expression cassettes, hSpCas9 and the chimeric guide RNA. The vector was digested using $B s m \mathrm{BI}$, and a pair of annealed oligos was subcloned into the sgRNA scaffold. Then, the cloned sgRNA lenti-CRISPR-v2 vector was sequence using the hU6 promoter primer. The lenti-CRISPR-v2 plasmid (with sgRNA cloned) was cotransfected into HEK293T cells with the packaging plasmids pVSVg (Addgene, 8454) and psPAX2 (Addgene, 12260). CMV-EGFP was used as a positive control for viral production. The lentivirus was concentrated by centrifugation at 20,000 $g$ for 2 hours at $4^{\circ} \mathrm{C}$. PC12 and 50B11 cells were infected by the concentrated lentivirus. They were selected 48 hours later by $2 \mu \mathrm{g} / \mathrm{mL}$ puromycin, incubated for another 48 hours, and then harvested to detect SIRT2 expression by Western blotting. sgRNA targeting 5'-GCGGAAGTCAGGGATTCCTG-3' showed optimal functionality with rat SIRT2. To rescue the Sirt2-KO PC12 and 50B11 cells, the plasmids pcDNA-SIRT2flag (WT), pcDNA-SIRT2-flag (H150Y, mutant) $(28,37)$, and an empty vector were used.

Immunoblotting and antibodies. Cells were washed twice with prechilled PBS and lysed with lysis buffer ( $150 \mathrm{mM} \mathrm{NaCl}, 50 \mathrm{mM}$ Tris, pH 8.0, 5 mM EDTA, 1.0\% Nonidet P-40, 0.1\% SDS, 0.5\% sodium deoxycholate), protease inhibitors (Sigma-Aldrich, catalog P8340), and phosphatase inhibitor cocktail (Roche, catalog 4906837001) and analyzed by SDS-PAGE. Antibodies used for immunoblotting were anti-SIRT2 (Cell Signaling Technology, catalog 12672S; Thermo Fisher, catalog PA5-20487; Proteintech, 19655-1-AP; 1:500), anti-Sirt1 (Cell Signaling Technology, catalog 8469S), anti-XPB (Cell Signaling Technology, catalog 8746), anti- $\alpha$-tubulin (Cell Signaling Technology, catalog 2125), anti-acetyl $\alpha$-tubulin (Lys 40) (Cell Signaling Technology, catalog 5335), anti-acetyl p53 (Lys373, Lys382) (Sigma-Aldrich, catalog 06-758), anti-GAPDH (Cell Signaling Technology, catalog 2118S), anti-vinculin (Sigma-Aldrich, catalog V4505, 1:2000), and anti-histone H1 (Santa Cruz Biotechnology, catalog 393358). All the antibodies were used at 1:1000 dilution unless otherwise indicated.

Dot blot. Forskolin-induced neuronally differentiated 50B11 cells were treated with $2 \mu \mathrm{g} / \mathrm{mL}$ cisplatin for 24 hours, and cells were then harvested at $4^{\circ} \mathrm{C}$, followed by DNA extraction and purification using the DNeasy Blood \& Tissue kit (Qiagen, catalog 69506). DNA-platinum adduct dot blotting was performed as described previously (80). One microgram of DNA in $2 \mu \mathrm{L}$ TE buffer (10 mM Tris- $\mathrm{HCl} \mathrm{pH} \mathrm{8.0,}$ $1 \mathrm{mM}$ EDTA) was spotted onto nylon membranes (Hybond-N; GE Healthcare, catalog RPN82N) and allowed to dry. After cross-linking by UV light, membranes were incubated in blocking solution (5\% milk in TBST, a mixture of Tris-buffered saline [TBS] and Polysorbate 20) for 1 hour, followed by incubation with anti-DNA/platinum adduct antibody (anti-GG, clone ICR4; EMD-Millipore, catalog MABE416) at 
$4^{\circ} \mathrm{C}$ on a shaker overnight. After washing the membrane 3 times with TBST for 10 minutes each, the membrane was incubated with secondary antibody (goat anti-rat IgG conjugated to HRP, Cell Signaling Technology, catalog 7707S) for 1 hour at room temperature. Then the membrane was washed again 3 times (10 minutes each), developed with Western Lighting Plus ECL (PerkinElmer, catalog 104001EA), and imaged in a ChemiDoc MP (Bio-Rad). In order to measure the total DNA loading, following brief washing with TBST, the membrane was immediately incubated in ethidium bromide/TBST solution for 30 minutes, followed by TBST washing and imaging as above. Dot blot densitometry analysis was performed using ImageJ.

Cell survival assay. Forty thousand PC12 or 50B11 (Sirt2-WT cell line and Sirt2-KO cell line with vector, Sirt2-KO/WT-Sirt2, or Sirt2$\mathrm{KO} / \mathrm{HY}$-Sirt2 rescue expression) cells/well were seeded in 6-well plates. After induction of differentiation for 24 hours $(49,81)$, cells were treated with varying concentrations of cisplatin for 48 hours. For SP treatment, differentiated 50B11 cells were pretreated with 10 $\mu \mathrm{M}$ SP for 1 hour followed by cisplatin treatment. For LLC and H1299 cells, 25,000 cells/well were plated, and cisplatin treatment lasted for 72 hours. At the end of the experiments, 6-well plates were kept on ice, and the number of live cells from each sample was determined using an Automated Cell Counter (Bio-Rad, TC20) after trypan blue staining. The survival fraction was calculated as the number of cells that survived after drug treatment normalized to the number of cells that survived after vehicle treatment times 100.

Cross-link repair efficiency assay. The luciferase activity was measured using the Dual-Luciferase Assay kit (Promega). Interstrand cross-linked reporter plasmid (pCMV-Luc XL) was prepared by incubating pCMV-Luc DNA $(0.2 \mathrm{mg} / \mathrm{mL})$ with $5 \mu \mathrm{M}$ cisplatin in TE buffer for 3 hours at $37^{\circ} \mathrm{C}$ in the dark. The reaction was stopped by $\mathrm{NaCl}$ (added to $0.5 \mathrm{M}$ ). Plasmid DNA was ethanol precipitated, washed in $70 \%$ ethanol, dried, and redissolved in TE buffer. PBS-incubated plasmid was used as a control. Cells were transfected with $1 \mathrm{ng}$ pCMV-Luc XL or control, $1 \mathrm{ng}$ TK-Renilla luciferase plasmid (internal control), and $1 \mu \mathrm{g}$ carrier DNA per well of a 6-well plate. The luciferase reporter assay was performed 24 hours after transfection. Firefly luciferase (CMV-Luc) activity was normalized to Renilla (TK-Luc) luciferase activity, and the ratio of normalized pCMV-Luc XL to control readings per sample was calculated as cross-link repair efficiency. XPA and C-XPA cell lines were used as negative and positive controls for the assay. The plasmids and cells were a gift from Lei Li (MD Anderson Cancer Center).

Statistics. Data are presented as mean \pm SEM and are representative data from 3 independent experiments, unless otherwise indicated, for all in vitro studies. Statistical analysis of the differences among groups was performed with Prism 6.0 (GraphPad) for Windows using 1-way ANOVA with Tukey's or Dunnett's post hoc analysis, 2-way ANOVA with Bonferroni's posttests, or 2-tailed Student's $t$ test. ${ }^{*} P<$ 0.05 was deemed statistically significant, ${ }^{* *} P<0.01$ highly significant, and ${ }^{* * *} P<0.001$ extremely significant.

Study approval. All procedures were approved by the University of Arkansas for Medical Sciences Institutional Animal Care and Use Committee (IACUC, Little Rock, Arkansas). All animal experiments were performed in accordance with NIH regulations on the use and care of experimental animals.

\section{Author contributions}

FX, MZ, WD, and SJ conceived and designed the experiments. MZ, WD, and SJ performed the experiments. FX, MZ, WD, SA, and SJ analyzed the data. SA, MZ, and FX wrote the manuscript. All authors reviewed and approved the manuscript for publication. The contributions of MZ, WD, and SA are equivalent, and the order of names was decided by seniority within the research group.

\section{Acknowledgments}

We gratefully acknowledge David Sinclair (Harvard Medical School) for providing essential reagents. This study was supported by NIH grants R01 CA188500 to FX and AC and R01 CA163838 to FX. We thank the University of Arkansas for Medical Sciences' Science Communication Office for editorial assistance during the preparation of this manuscript.

Address correspondence to: Fen Xia, Department of Radiation Oncology, 4301 West Markham, Slot 771, Little Rock, Arkansas 72205, USA. Phone: 501.526.7458; Email: fxia@uams.edu.
1. Cioroiu C, Weimer LH. Update on chemotherapy-induced peripheral neuropathy. Curr Neurol Neurosci Rep. 2017;17(6):47.

2. Han Y, Smith MT. Pathobiology of cancer chemotherapy-induced peripheral neuropathy (CIPN). Front Pharmacol. 2013;4:156.

3. Kandula T, et al. Neurophysiological and clinical outcomes in chemotherapy-induced neuropathy in cancer. Clin Neurophysiol. 2017;128(7):1166-1175.

4. Aloe L, Manni L, Properzi F, De Santis S, Fiore M. Evidence that nerve growth factor promotes the recovery of peripheral neuropathy induced in mice by cisplatin: behavioral, structural and biochemical analysis. Auton Neurosci. 2000;86(1-2):84-93.

5. Boogerd W, ten Bokkel Huinink WW, Dalesio O, Hoppenbrouwers WJ, van der Sande JJ. Cisplatin induced neuropathy: central, peripheral and autonomic nerve involvement. J Neurooncol. 1990;9(3):255-263.

6. Cata JP, Weng HR, Lee BN, Reuben JM, Dougherty PM. Clinical and experimental findings in humans and animals with chemotherapy-in- duced peripheral neuropathy. Minerva Anestesiol. 2006;72(3):151-169.

7. Hu LY, et al. Triggering receptor expressed on myeloid cells 2 (TREM2) dependent microglial activation promotes cisplatin-induced peripheral neuropathy in mice. Brain Behav Immun. 2018;68:132-145.

8. Imai S, et al. Taxanes and platinum derivatives impair Schwann cells via distinct mechanisms. Sci Rep. 2017;7(1):5947.

9. Kerckhove N, Collin A, Condé S, Chaleteix C, Pezet D, Balayssac D. Long-term effects, pathophysiological mechanisms, and risk factors of chemotherapy-induced peripheral neuropathies: a comprehensive literature review. Front Pharmacol. 2017;8:86.

10. Boulikas T, Vougiouka M. Cisplatin and platinum drugs at the molecular level. (Review). Oncol Rep. 2003;10(6):1663-1682.

11. Englander EW. DNA damage response in peripheral nervous system: coping with cancer therapy-induced DNA lesions. DNA Repair (Amst).
2013;12(8):685-690.

12. McDonald ES, Randon KR, Knight A, Windebank AJ. Cisplatin preferentially binds to DNA in dorsal root ganglion neurons in vitro and in vivo: a potential mechanism for neurotoxicity. Neurobiol Dis. 2005;18(2):305-313.

13. Dzagnidze A, et al. Repair capacity for platinum-DNA adducts determines the severity of cisplatin-induced peripheral neuropathy. J Neurosci. 2007;27(35):9451-9457.

14. Furuta T, Ueda T, Aune G, Sarasin A, Kraemer KH, Pommier Y. Transcription-coupled nucleotide excision repair as a determinant of cisplatin sensitivity of human cells. Cancer Res. 2002;62(17):4899-4902.

15. Welsh C, Day R, McGurk C, Masters JR, Wood RD, Köberle B. Reduced levels of XPA, ERCC1 and XPF DNA repair proteins in testis tumor cell lines. Int J Cancer. 2004;110(3):352-361.

16. Wood $\mathrm{RD}$, et al. DNA damage recognition and nucleotide excision repair in mammalian cells. Cold Spring Harb Symp Quant Biol. 2000;65:173-182. 
17. Adar S, Hu J, Lieb JD, Sancar A. Genome-wide kinetics of DNA excision repair in relation to chromatin state and mutagenesis. Proc Natl Acad Sci U S A. 2016;113(15):E2124-E2133.

18. Choi JY, Park JM, Yi JM, Leem SH, Kang TH. Enhanced nucleotide excision repair capacity in lung cancer cells by preconditioning with DNA-damaging agents. Oncotarget. 2015;6(26):22575-22586.

19. Madabhushi R, Pan L, Tsai LH. DNA damage and its links to neurodegeneration. Neuron. 2014;83(2):266-282.

20. Wei L, et al. DNA damage during the GO/G1 phase triggers RNA-templated, Cockayne syndrome B-dependent homologous recombination. Proc Natl Acad Sci U S A . 2015;112(27):E3495-E3504.

21. Berta T, Qadri Y, Tan PH, Ji RR. Targeting dorsal root ganglia and primary sensory neurons for the treatment of chronic pain. Expert Opin Ther Targets. 2017;21(7):695-703

22. Guha D, Shamji MF. The dorsal root ganglion in the pathogenesis of chronic neuropathic pain. Neurosurgery. 2016;63 suppl 1:118-126.

23. Zimmermann M. Pathobiology of neuropathic pain. Eur J Pharmacol. 2001;429(1-3):23-37.

24. Stewart DJ, et al. Human central nervous system distribution of cis-diamminedichloroplatinum and use as a radiosensitizer in malignant brain tumors. Cancer Res. 1982;42(6):2474-2479.

25. Ta LE, Espeset L, Podratz J, Windebank AJ. Neurotoxicity of oxaliplatin and cisplatin for dorsal root ganglion neurons correlates with platinum-DNA binding. Neurotoxicology. 2006;27(6):992-1002

26. Beirowski B, et al. Sir-two-homolog 2 (Sirt2) modulates peripheral myelination through polarity protein Par-3/atypical protein kinase C (aPKC) signaling. Proc Natl Acad Sci U S A. 2011;108(43):E952-E961.

27. Li S, et al. MicroRNA-7 inhibits neuronal apoptosis in a cellular Parkinson's disease model by targeting Bax and Sirt2. Am J Transl Res. 2016;8(2):993-1004.

28. Park SH, et al. SIRT2-mediated deacetylation and tetramerization of pyruvate kinase directs glycolysis and tumor growth. Cancer Res. 2016;76(13):3802-3812.

29. Park SH, et al. SIRT2 is a tumor suppressor that connects aging, acetylome, cell cycle signaling, and carcinogenesis. Transl Cancer Res. 2012;1(1):15-21.

30. Singh P, Hanson PS, Morris CM. Sirtuin-2 protects neural cells from oxidative stress and is elevated in neurodegeneration. Parkinsons Dis. 2017;2017:2643587.

31. Finnin MS, Donigian JR, Pavletich NP. Structure of the histone deacetylase SIRT2. Nat Struct Biol. 2001;8(7):621-625.

32. North BJ, Marshall BL, Borra MT, Denu JM, Verdin E. The human Sir2 ortholog, SIRT2, is an $\mathrm{NAD}^{+}$-dependent tubulin deacetylase. Mol Cell. 2003;11(2):437-444.

33. Wang F, Nguyen M, Qin FX, Tong Q. SIRT2 deacetylates FOXO3a in response to oxidative stress and caloric restriction. Aging Cell. 2007;6(4):505-514.

34. Flick F, Lüscher B. Regulation of sirtuin function by posttranslational modifications. Front Phar- macol. 2012;3:29.

35. Rack JG, VanLinden MR, Lutter T, Aasland R, Ziegler M. Constitutive nuclear localization of an alternatively spliced sirtuin-2 isoform. J Mol Biol. 2014;426(8):1677-1691.

36. North BJ, et al. SIRT2 induces the checkpoint kinase BubR1 to increase lifespan. EMBO J. 2014;33(13):1438-1453.

37. Zhang H, et al. SIRT2 directs the replication stress response through CDK9 deacetylation. Proc Natl Acad Sci U S A. 2013;110(33):13546-13551.

38. Pan Y, et al. Resveratrol exerts antioxidant effects by activating SIRT2 to deacetylate Prx1. Biochemistry. 2017;56(48):6325-6328.

39. Suzuki K, Koike T. Resveratrol abolishes resistance to axonal degeneration in slow Wallerian degeneration (WldS) mice: activation of SIRT2 an NAD-dependent tubulin deacetylase. Biochem Biophys Res Commun. 2007;359(3):665-671.

40. Trammell SA, et al. Nicotinamide riboside is uniquely and orally bioavailable in mice and humans. Nat Commun. 2016;7:12948.

41. Hamity MV, White SR, Walder RY, Schmidt MS, Brenner C, Hammond DL. Nicotinamide riboside, a form of vitamin $\mathrm{B} 3$ and $\mathrm{NAD}^{+}$precursor, relieves the nociceptive and aversive dimensions of paclitaxel-induced peripheral neuropathy in female rats. Pain. 2017;158(5):962-972.

42. Trammell SA, et al. Nicotinamide riboside opposes type 2 diabetes and neuropathy in mice. $S c i$ Rep. 2016;6:26933.

43. Boehmerle W, Huehnchen P, Peruzzaro S, Balkaya M, Endres M. Electrophysiological, behavioral and histological characterization of paclitaxel, cisplatin, vincristine and bortezomib-induced neuropathy in C57BL/6 mice. Sci Rep. 2014;4:6370.

44. Mao-Ying QL, et al. The anti-diabetic drug metformin protects against chemotherapy-induced peripheral neuropathy in a mouse model. PLoS One. 2014;9(6):e100701.

45. Ta LE, et al. A novel and selective poly (ADP-ribose) polymerase inhibitor ameliorates chemotherapy-induced painful neuropathy. PLoS One. 2013;8(1):e54161

46. Lin $\mathrm{H}, \mathrm{Heo} \mathrm{BH}$, Yoon $\mathrm{MH}$. A new rat model of cisplatin-induced neuropathic pain. Korean J Pain. 2015;28(4):236-243.

47. Ta LE, Bieber AJ, Carlton SM, Loprinzi CL, Low PA, Windebank AJ. Transient Receptor Potential Vanilloid 1 is essential for cisplatin-induced heat hyperalgesia in mice. Mol Pain. 2010;6:15.

48. Ta LE, Low PA, Windebank AJ. Mice with cisplatin and oxaliplatin-induced painful neuropathy develop distinct early responses to thermal stimuli. Mol Pain. 2009;5:9.

49. Chen W, Mi R, Haughey N, Oz M, Höke A. Immortalization and characterization of a nociceptive dorsal root ganglion sensory neuronal line. J Peripher Nerv Syst. 2007;12(2):121-130.

50. Zheng H, et al. Nucleotide excision repair- and polymerase eta-mediated error-prone removal of mitomycin C interstrand cross-links. Mol Cell Biol. 2003;23(2):754-761.

51. Li L, Lu X, Peterson CA, Legerski RJ. An interaction between the DNA repair factor XPA and replication protein A appears essential for nucleotide excision repair. Mol Cell Biol.
1995;15(10):5396-5402.

52. Li L, Peterson CA, Lu X, Legerski RJ. Mutations in XPA that prevent association with ERCC1 are defective in nucleotide excision repair. Mol Cell Biol. 1995;15(4):1993-1998.

53. Sanjana NE, Shalem O, Zhang F. Improved vectors and genome-wide libraries for CRISPR screening. Nat Methods. 2014;11(8):783-784.

54. Shalem O, et al. Genome-scale CRISPR-Cas9 knockout screening in human cells. Science. 2014;343(6166):84-87.

55. Wang G, et al. SIRT1 involved in the regulation of alternative splicing affects the DNA damage response in neural stem cells. Cell Physiol Biochem. 2018;48(2):657-669.

56. Wang RH, et al. Impaired DNA damage response, genome instability, and tumorigenesis in SIRT1 mutant mice. Cancer Cell. 2008;14(4):312-323.

57. Zhao M, et al. PCAF/GCN5-mediated acetylation of RPA1 promotes nucleotide excision repair. Cell Rep. 2017;20(9):1997-2009.

58. Vaur $\mathrm{P}$, et al. Nicotinamide riboside, a form of vitamin $B_{3}$, protects against excitotoxicity-induced axonal degeneration. FASEB J. 2017;31(12):5440-5452.

59. Alekseev S, Ayadi M, Brino L, Egly JM, Larsen AK, Coin F. A small molecule screen identifies an inhibitor of DNA repair inducing the degradation of TFIIH and the chemosensitization of tumor cells to platinum. Chem Biol. 2014;21(3):398-407.

60. Cea M, et al. Evidence for a role of the histone deacetylase SIRT6 in DNA damage response of multiple myeloma cells. Blood. 2016;127(9):1138-1150.

61. Chen W, et al. Sirt6 promotes DNA end joining in iPSCs derived from old mice. Cell Rep. 2017;18(12):2880-2892.

62. $\mathrm{Li} \mathrm{L}$, et al. SIRT7 is a histone desuccinylase that functionally links to chromatin compaction and genome stability. Nat Commun. 2016;7:12235.

63. Vazquez BN, et al. SIRT7 promotes genome integrity and modulates non-homologous end joining DNA repair. EMBO J. 2016;35(14):1488-1503.

64. Tomaszewski A, Büsselberg D. Cisplatin modulates voltage gated channel currents of dorsal root ganglion neurons of rats. Neurotoxicology. 2007;28(1):49-58.

65. Carozzi VA, Canta A, Chiorazzi A. Chemotherapy-induced peripheral neuropathy: What do we know about mechanisms? Neurosci Lett. 2015;596:90-107.

66. Gulec M, et al. Mirtazapine protects against cisplatin-induced oxidative stress and DNA damage in the rat brain. Psychiatry Clin Neurosci. 2013;67(1):50-58

67. Jiang Y, Guo C, Vasko MR, Kelley MR. Implications of apurinic/apyrimidinic endonuclease in reactive oxygen signaling response after cisplatin treatment of dorsal root ganglion neurons. Cancer Res. 2008;68(15):6425-6434.

68. Piccolini VM, Bottone MG, Bottiroli G, De Pascali SA, Fanizzi FP, Bernocchi G. Platinum drugs and neurotoxicity: effects on intracellular calcium homeostasis. Cell Biol Toxicol. 2013;29(5):339-353.

69. Waseem M, Parvez S. Mitochondrial dysfunction mediated cisplatin induced toxicity: modulatory role of curcumin. Food Chem Toxicol. 2013;53:334-342. 
70. Yang X, et al. Sirtuin 2 regulates cellular iron homeostasis via deacetylation of transcription factor NRF2. J Clin Invest. 2017;127(4):1505-1516.

71. Inoue T, et al. SIRT2, a tubulin deacetylase, acts to block the entry to chromosome condensation in response to mitotic stress. Oncogene. 2007;26(7):945-957.

72. Rumpf T, et al. Selective Sirt2 inhibition by ligand-induced rearrangement of the active site. Nat Commun. 2015;6:6263.

73. Skoge RH, Dölle C, Ziegler M. Regulation of SIRT2-dependent $\alpha$-tubulin deacetylation by cellular NAD levels. DNA Repair (Amst). 2014;23:33-38.

74. Abdel-Salam OME, Baiuomy AR, Nada SA. Effect of spironolactone on pain responses in mice.
EXCLIJ. 2010;9:46-57.

75. Lewin GR, Moshourab R. Mechanosensation and pain. J Neurobiol. 2004;61(1):30-44.

76. Gamble GD, Milne RJ. Repeated exposure to sham testing procedures reduces reflex withdrawal and hot-plate latencies: attenuation of tonic descending inhibition? Neurosci Lett. 1989;96(3):312-317.

77. Plone MA, Emerich DF, Lindner MD. Individual differences in the hotplate test and effects of habituation on sensitivity to morphine. Pain. 1996;66(2-3):265-270.

78. Aydın TH, Can ÖD, Demir Özkay Ü, Turan N. Effect of subacute agomelatine treatment on painful diabetic neuropathy: involvement of catecholaminergic mechanisms. Fundam Clin Pharmacol. 2016;30(6):549-567.

79. Mert T, Gunes Y, Gunay I. Comparison of actions of systemically and locally administrated local anaesthetics in diabetic rats with painful neuropathy. Fundam Clin Pharmacol. 2013;27(2):161-168.

80. Zhu J, et al. Ethoxyquin provides neuroprotection against cisplatin-induced neurotoxicity. Sci Rep. 2016;6:28861.

81. Das KP, Freudenrich TM, Mundy WR. Assessment of PC12 cell differentiation and neurite growth: a comparison of morphological and neurochemical measures. Neurotoxicol Teratol. 2004;26(3):397-406. 